UDC 578.24

\title{
VENEZUELAN EQUINE ENCEPHALITIS VIRUS CAPABLE OF NON-CYTOPATHIC REPLICATION: VIRUS PRODUCTION AND GROWTH IN CELLS WITH VARYING DEGREES OF RESISTANCE TO INFECTION
}

\author{
Keyer V.V., Baltabekova A.Zh., Mahatova A.S., Shustov A.V. \\ National Center for Biotechnology \\ 13/5, Korgalzhyn road, Astana, 010000, Kazakhstan \\ keerv@mail.ru
}

\section{ABSTRACT}

The model alphavirus Venezuelan equine encephalitis virus (VEE) exhibits non-cytopathic replication in cell cultures. In this study, the ability of VEE to infect cells of different types was investigated. Noncytopathic VEE was capable of a persistent infection in cultures of BHK-21 fibroblasts, HEK293T epithelial cells, and X63-Ag8.653 plasma cells, which all show varying abilities to suppress viral replication through an interferon (IFN)-dependent mechanism. The ability of VEE to infect a broad range of cell types and replicate without inducing cell death makes this virus an attractive vector for eukaryotic expression. In particular, persistent VEE infection was demonstrated in the myeloid cell line X63, which is capable of an IFN-induced antiviral state. This observation underscores the potential application of VEE-based vectors to drive recombinant protein expression in highly productive plasmacytoma cells.

Key words: alphavirus, RNA-containing virus, Venezuelan equine encephalitis virus, non-cytopathic replication, growth curve.

\section{INTRODUCTION}

Expanding needs of biomedical research and industrial purposes pose immense pressure for an improvement in technologies for expression of recombinant protein in in vitro cultured cells from higher eukaryotes. In particular, the eukaryotic expression is required to produce proteins with high added value, such as biopharmaceuticals (proteins with pharmacological activity: recombinant monoclonal antibodies, erythropoietin, blood clotting factors, etc.). Currently $\sim 50 \%$ of biopharmaceuticals are produced by expression in mammalian cell cultures. The expression in an eukaryotic system is needed when a target protein does not acquire a correct spatial fold in other systems, and when there is a need to provide post-translational modifications, primarily glycosylation. Two strategies are commonly used to achieve the recombinant expression in the cell cultures: one strategy involves creating of genetically stable cell lines in which a gene of interest is integrated into chromosomal DNA of the host cells. The second strategy (transient expression) utilizes so-called mobilizable (not integrated into the genome) vectors. The biotechnology industry is currently dominated by the first strategy (genetically stable producer lines) despite that this strategy requires large initial costs at the stage of obtaining and selecting a clone with a high expression. One drawback of this strategy is a lengthy process of obtaining a highly productive line [1]. The transient expression greatly speeds up a production of the producer lines; this approach is effective when a rapid production of a product is needed, or a high-throughput screening of different proteins is planned, for example to test several variants for a biological activity. Plasmids are a predominate type of vectors for transient expression, including in cell cultures. Commonly the eukaryotic plasmids carry origins of replication (Ori) of DNA-containing viruses. The presence of viral Ori regions provides long-term persistence of plasmids in nuclei of transformed cells [2]. At the same time, problems persist with the plasmid-driven transient expression, which are low levels of expression and a pronounced temporal (transient) nature of the expression. A cell culture transformed with a plasmid usually produces a product at the maximum level for less than 1 week and then the expression decreases. An alternative type of vectors has been exploited which represent viruses or fragments of viral genomes capable of autonomous replication (replicons) within a cell. Still most popular viral vectors for mammalian cell cultures (excluding use in a gene therapy) are adenoviruses [3], retroviruses [4], lentiviruses [5], poxviruses (Vaccinia virus) [6] or baculoviruses (viruses of insects) into the genomes of which mammalian gene cassettes have been engineered [7]. The above examples refer to DNA-containing viruses or to RNA-viruses for which there is a DNA stage (reverse transcription) in their life cycle. Different and highly efficient protein expression systems have been created utilizing RNA-containing viruses. 
Genomes of RNA-viruses exist only in the form of RNA, i.e. all copying of viral sequences is carried out by RNAdependent RNA polymerase.

Alphaviruses are representatives of the genus Alphavirus, family Togaviridae, and they are attractive vectors for heterologous expression [8]. In this genus, there are 35 species that are grouped into subgroups according to their geographical distribution. The subgroups are named after their most studies species, for example, the Semliki forest virus (SFV) subgroup, the Sindbis virus (SIN) subgroup, and the Venezuelan equine encephalitis virus (VEE) subgroup. Among alphaviruses used as vectors in laboratory and even in industry are the SFV [9, 10], SIN [11] and VEE [12, 13]. Intracellular replication of alphaviruses is accompanied with a synthesis of large amounts of viral proteins. It was demonstrated, that production from a foreign gene integrated into a genome of an alphavirus may be $25 \%$ of a total cellular protein [14].

However, there exist obstacles to a practical use of alphaviruses as vectors. The first obstacle is a profound cytopathicity of wild-type alphaviruses. A cytopathic effect (CPE) is a characteristic phenomenon for all natural strains of alphaviruses. The CPE leads to death of the infected cell culture within 24-48 hours after the infection. The second obstacle is that majority of cell lines (for example, all primary cultures) respond to the infection with alphaviruses by activating mechanisms of innate cellular immunity, of which the most important for suppressing replication of VEE is an establishment of a so-called "antiviral state". The antiviral state occurs under influence of interferons (IFNs) and in cells with unimpaired natural mechanisms of antiviral response, the IFN-induced effects significantly suppress viral replication.

This article describes a creation of a VEE-derived virus which differs from the parental strain by a noncytopathic replication. Characteristics of the virus' ability to growth in various cell cultures (growth curves) were studied. Three cell lines differing in ability to suppress viral replication were used in the study, among them BHK21, HEK293T and X63-AG8.653. The line HEK293T is routinely used in a biopharmaceutical industry and X63 cells have high prospects of utilization as efficient producers apart from their use to construct murine hybridomas. The non-cytopathic VEE infects all cell lines and establishes persistent infection, although virus yields strongly depend on a type of the cells.

\section{MATERIALS AND METHODS}

\section{Producing genetically engineered constructs}

Methods of genetic engineering are described in our previous article [15]. Assembled constructs are the E.coli plasmids with inserts of cDNA copies of a full-length VEE genome. The non-cytopathic VEE described in this article was constructed by introducing mutations into known locations of the genome of VEE strain TC83 which was constructed using described from de novo assembly and described in [15]. In addition to the mutations introduced to reduce the CPE during intracellular replication, the genome of the created virus carries synthetic genes encoding puromycin acetyltransferase (enzyme which provides resistance to the antibiotic puromycin) and green fluorescent protein (GFP).

The changes to the VEE genes described in this article were made by PCR-mediated mutagenesis; cloning of the resulting PCR fragment into starting plasmids was done using restriction sites and a standard restriction-ligation process. To perform the PCR, a high-precision polymerase Phusion (Thermo Scientific) was used. All PCR products passed an intermediate cloning into a vector pGEM-T (Promega) for sequencing. All resulting constructs were bidirectionally sequenced to confirm absence of unwanted nucleotide substitutions. The genome VEE(L.Cm).Pac2A-GFP which was used in this study was deposited to the GenBank database: MF136452.

\section{In vitro transcription of virus genomic RNA}

Plasmid carrying a cDNA copy of the VEE genome was converted into a linear form by digestion with MluI. The linearized plasmid was extracted with phenol-chloroform, precipitated with EtOH and dissolved in water to a final concentration $0.2 \mu \mathrm{g} / \mu \mathrm{l}$.

Reaction mixtures for in vitro transcription were prepared by mixing $12.5 \mu$ of water, $10 \mu 1$ of $5 \mathrm{X}$ SP6 buffer, $5 \mu \mathrm{l}$ of a solution of dithiothreitol (10 mM DTT in water), $2.5 \mu \mathrm{l}$ of a solution of synthetic analogue of cap structure $\left(\mathrm{m} 7 \mathrm{G}\left(5^{\prime}\right) \mathrm{ppp}\left(5^{\prime}\right) \mathrm{G}\right)(10 \mathrm{mM}$ in water), $10 \mu \mathrm{l}$ of $10 \mathrm{mM}$ ribonucleoside triphosphates (rNTP) and $10 \mu \mathrm{l}$ of linearized DNA $(200 \mathrm{ng} / \mu \mathrm{l})$. To the mixture were added $1 \mu \mathrm{l}$ of RNase inhibitor (RiboLock, ThermoFisher Scientific) and 2.5 $\mu$ of SP6 RNA polymerase (ThermoFisher Scientific). The mixture was incubated at $41^{\circ} \mathrm{C}$ for 1 hour. Quality of the RNA synthesis was monitored by electrophoresis in $1 \%$ agarose.

\section{Cell cultures}

Baby hamster kidney (BHK-21) cells, human embryonic kidney (HEK293T) cells were grown in DMEM supplemented with $10 \%$ fetal calf serum (FBS), $1 \%$ penicillin/streptomycin, $10 \mathrm{mM} \mathrm{L-glutamine}$ at $37^{\circ} \mathrm{C}$ in an atmosphere of $5 \% \mathrm{CO}_{2}$. X63-AG8.653 cells were grown in RPMI 1640 medium with addition of 10\% FBS, 1\% penicillin/streptomycin, $10 \mathrm{mM} \mathrm{L-glutamine,} \mathrm{at} 37^{\circ} \mathrm{C}$ in $5 \% \mathrm{CO}_{2}$

\section{Transfection of cell cultures with RNA}

For transfections, cultures grown in 6 -well plates to $70-90 \%$ confluence $\left(\sim 5 \times 10^{\wedge} 5\right.$ cells $)$ were used. Transfections were performed using cationic liposomes (Lipofectamine 2000, Life Technologies). Portions of $250 \mu 1$ of Opti-MEM medium (Life Technologies) were placed into two tubes and $10 \mu$ of the synthetic RNA was added to 
one tube, $10 \mu \mathrm{l}$ of Lipofectamine 2000 was added to the second tube. Tubes were incubated for 5 minutes at room temperature and then the contents of the two tubes were combined. The combined mixture was incubated for additional 20 minutes at room temperature. Then the mixture was introduced into a well (of a 6 well plate) to coat a monolayer of cells previously washed with PBS. The cells were incubated in the presence of RNA and lipofectamine for 4 hours. The Opti-MEM medium was then removed from the monolayer and $2 \mathrm{ml}$ of complete growth medium was added into the wells. The virus-containing culture medium was harvested from the wells 48 hours after the transfection and stored at $-80^{\circ} \mathrm{C}$.

\section{Determination of virus titers and production of growth curves}

Virus titers were determined according to the Reed-Mench method by infecting BHK-21 cells grown in 96well plates with serial dilutions of the virus preparation. In 96 -well plates BHK-21 cells were seeded, $3.75 \times 10^{\wedge} 4$ cells per well. The plates were incubated for 4 hours at $37^{\circ} \mathrm{C}$ in a $\mathrm{CO}_{2}$ chamber. Dilutions of the virus-containing media were prepared, starting from $1: 10$ to $1: 10^{\wedge} 8$. Dilutions were prepared in phosphate buffered saline (PBS, Sigma D8537) supplemented with $1 \%$ horse serum. The medium was removed from the wells (of 96-well plates) and aliquots $(100 \mu \mathrm{l})$ of the corresponding dilution were introduced into each well (12 wells in one row were infected with the same dilution; rows A-H pate were infected with dilutions 1:10-1:10-8). Plates were incubated for 1 hour in a CO2 incubator; every 15 minutes the plates were shaken to allow uniform mixing in the wells. Then, the liquid was removed from the wells and the wells were immediately filled with a complete growth medium $(150 \mu 1$ per well). Infected cultures were incubated for 24 hours. GFP fluorescence in infected cells was visualized under a fluorescent microscope. The well was considered positive if at least a part of the cells showed fluorescence; if all cells in the well did not show GFP fluorescence, the well was considered negative. The results of titration were calculated as described in [16].

\section{Infection of cell cultures BHK-21, HEK293T and X63-AG8.653}

Cultures BHK-21, HEK293T, X63-AG8.653 were obtained from a collection of the National Center for Biotechnology (Astana). Cells were plated in $10 \mathrm{~cm}$ petri dishes in an amount of $2 \times 10^{\wedge} 6$ cells per dish. After the cells attached (4-24 hours), the cultures were infected with a virus with a multiplicity of infection MOI=1. For infection, medium was removed from the monolayers and $2 \mathrm{ml}$ of viral inoculum were added to the dishes. The infectious inoculum is a medium harvested from BHK-21 cells transfected with the viral RNA. The inoculum was diluted in PBS ( $+1 \%$ horse serum) so that in $2 \mathrm{ml}$ of the inoculum there are $2 * 10^{\wedge} 6$ focus-forming units (FFU) of the virus. The dishes were incubated for 1 hour. Then liquid was removed from the dishes and $10 \mathrm{ml}$ of complete growth medium was added to all dishes. The infected cultures were incubated at $37^{\circ} \mathrm{C}$ with complete changes of medium every 24 hours. Samples of culture mediums from infected monolayers were collected every 24 hours, upon collection of samples the mediums in the dishes were completely replaced with a fresh medium (10 ml). The collected samples were stored at $-80^{\circ} \mathrm{C}$ until determination of titers.

\section{RESULTS AND DISCUSSION}

\section{Mutations reducing cytopathicity}

The molecular clone of VEE strain TC-83 (Genbank L01443) was used as the starting viral genome. This molecular clone is an E.coli plasmid that contains, as an insert, a complementary DNA (cDNA) copy of the fulllength VEE genome ( 11,000 nt). The viral cDNA was assembled from synthetic DNA fragments. The original plasmid in this article is named pVEE/TC-83.

The wild-type virus (strain TC-83) has a strong cytopathic effect (CPE) during replication in cell cultures. Death of infected cells occurs within 24-36 hours after infection with the wild-type virus.

In order to reduce the CPE, attenuating mutations were engineered into a genome of the VEE. The mutations were previously described in $[17,18]$. One mutation was introduced into a gene for non-structural protein nsP2 which is a component of the viral replicative complex. This is a single nucleotide change $\mathrm{T}_{3865} \rightarrow \mathrm{A}(\mathrm{Gln} 739 \rightarrow \mathrm{Leu})$ [17]. This mutation is found in the autonomously replicating fragments of VEE genome (replicons) adapted to persistence (prolonged non-cytopathic replication) in BHK-21 culture. Replicons are long fragments of viral RNA which are capable of self-supported replication in cytoplasm although incapable of infectious cell-to-cell propagation because the replicons have no viral structural proteins (i.e. proteins involved in a formation of virions). It was shown that the amino acid replacement $\mathrm{Gln}_{739}->$ Leu in nsP2 decreases a rate of replication of viral RNA. This decrease is most likely a cause of a lower degree of the CPE of the mutant replicons [17].

The change $\mathrm{T}_{3865} \rightarrow \mathrm{A}$ was introduced into the genome of the wild-type virus using a two-round PCR. Primers used in the PCR are listed in Table 1 (No. 1-4). A template in the PCR was a plasmid pVEE/TC-83. During the first (I) round amplification, PCR reactions were performed in two separate tubes with primers nsp2S_KpnI+nsp2mutAS in one tube (product length $527 \mathrm{bp}$ ) and nsp2mutS+nsp2AS_BsiW in a second tube (113 bp product). Purified products $(100 \mathrm{ng})$ of the round I amplification were combined and the mixture was used in a second (II) round amplification with primers nsp2S_KpnI+nsp2AS_BsiW. This resulted in a fused PCR product (615 bp) which was cloned into pGEM-T vector. Upon sequencing the insert was excised using restriction enzymes KpnI and BsiWI. The same restriction sites were used to clone the mutated fragment back into the original plasmid pVEE/TC-83. The resulting plasmid was named $\mathrm{pVEE}(\mathrm{L})$. 
An analysis of published literature shows that unlike its replicons, the virus (VEE) which is capable of infectious dissemination has a strong CPE even if it bears the attenuating mutation $\mathrm{T}_{3865} \rightarrow \mathrm{A}$ in the nsP2. Full-length genomes of alphaviruses differ from replicons in that they have genes of structural proteins. It was shown that the CPE in New World alphaviruses (to which VEE also belongs), is not only modulated by mutations in non-structural proteins, but to major extent is caused by a suppression of translation of cellular RNAs which occurs in a presence of structural proteins. The inhibition of translation occurs with a participation of a capsid protein (protein C), and an effect of this mechanism extends only to cellular mRNAs, but not to viral RNAs. It turned out that the capsid protein has other functions besides a packaging of the viral genome $[18,19]$. In an infected cell, the capsid protein binds to nuclear transport proteins and blocks nuclear pore complex (NPC), thus preventing of export of mRNAs from the cell nucleus to cytoplasm and suppressing the translation of cellular proteins. Since viral RNAs are produced in the cytoplasm, their translation is not inhibited.

In order to produce a mutant of alphavirus with a non-cytopathic replication, which retains ability for infectious propagation in cell cultures we devised to circumvent a pathogenesis mechanism as described in [20]. For this purpose clusters of replacement mutations were engineered into the nuclear localization signal (NLS) and the supraphysiological nuclear export signal (supraNES) in the capsid protein. The two signals (NSL and supraNES) are present in the capsid proteins of a variety of circulating strains of New World alphaviruses, and the wild-type capsid protein is believed to play a major role in the molecular mechanism of blocking of the nuclear pore [20]. Mutations to the $\mathrm{C}$ gene similar to those described in [18] were devised. To make a mutant, the PCR with primers No. 5-9 (table 1) was used.

Table 1. Primers used to produce mutations in nsP2 and capsid proteins of VEE to reduce the cytopathic effect

\begin{tabular}{|c|l|l|c|}
\hline No. & \multicolumn{1}{|c|}{ Name } & \multicolumn{1}{|c|}{ Sequence $^{1}$} & Length, nt \\
\hline 1 & nsp2S_KpnI & TCGCAGGTACCCACAACTGCCTCGG & 25 \\
\hline 2 & nsp2mutAS & AAAACTTGAAC(A)GCCGCGCTATAGC & 25 \\
\hline 3 & nsp2mutS & GCTATAGCGCGGC(T)GTTCAAGTTTTC & 26 \\
\hline 4 & nsp2AS_BsiW & TTGTGCGTACGGGCCTTGCGATCGT & 25 \\
\hline 5 & St_PspOM & AGAGGGGCCCCTATAACTCTCTACG & 25 \\
\hline 6 & CAs_Bgl & (CCGCCTCC)CCGGCGTTGCTTGAACGTC & 34 \\
\hline 7 & CSe1 & GCCATCCGCT(GCAGCT)CCGAAGAAGGAGGCCTCG & 42 \\
\hline 8 & CSe2 & $\begin{array}{l}\text { ACGCCGG(GGAGGCGGTGGC)GAGGGGCCATCCGCT( } \\
\text { GCAGCT)CC }\end{array}$ & 35 \\
\hline 9 & AS_Xma & GGCATCTAGAAGCTGCTTCCAGCAGCTCATCGTAG & and which are \\
\hline $\begin{array}{l}\text { Note: } \\
\text { mutations. }\end{array}$
\end{tabular}

A PCR was performed with primers CSe1+AS_XmaXba in the 1-st round and CSe2+AS_XmaXba in the 2-nd round. The pVEE/TC-83 plasmid was used as a template in the 1-st round, and a product was used as a template in the 2-nd round. Length of the resulting PCR product is $830 \mathrm{bp}$. In a different PCR, amplification was performed with primers St_PspOM+CAs_Bgl with the pVEE/TC-83 as a template (product length 1093 bp). Further, the purified fragments were mixed $(830+1093)$ and the mixture was used as template in a PCR with primers St_PspOM+CAs_Bgl. A fusion fragment (1908 bp) was obtained and cloned into the pGEM-T. Sequencingconfirmed inserts were excised by PspOMI and AflII sites and cloned into the pVEE(L) plasmids using the indicated sites. The resulting plasmid was named pVEE(L)-Cm.

A comparison of amino acid sequences of the wild-type VEE capsid protein (strain TC-83) and the mutant capsid protein $(\mathrm{Cm})$ is shown in figure 1 . 


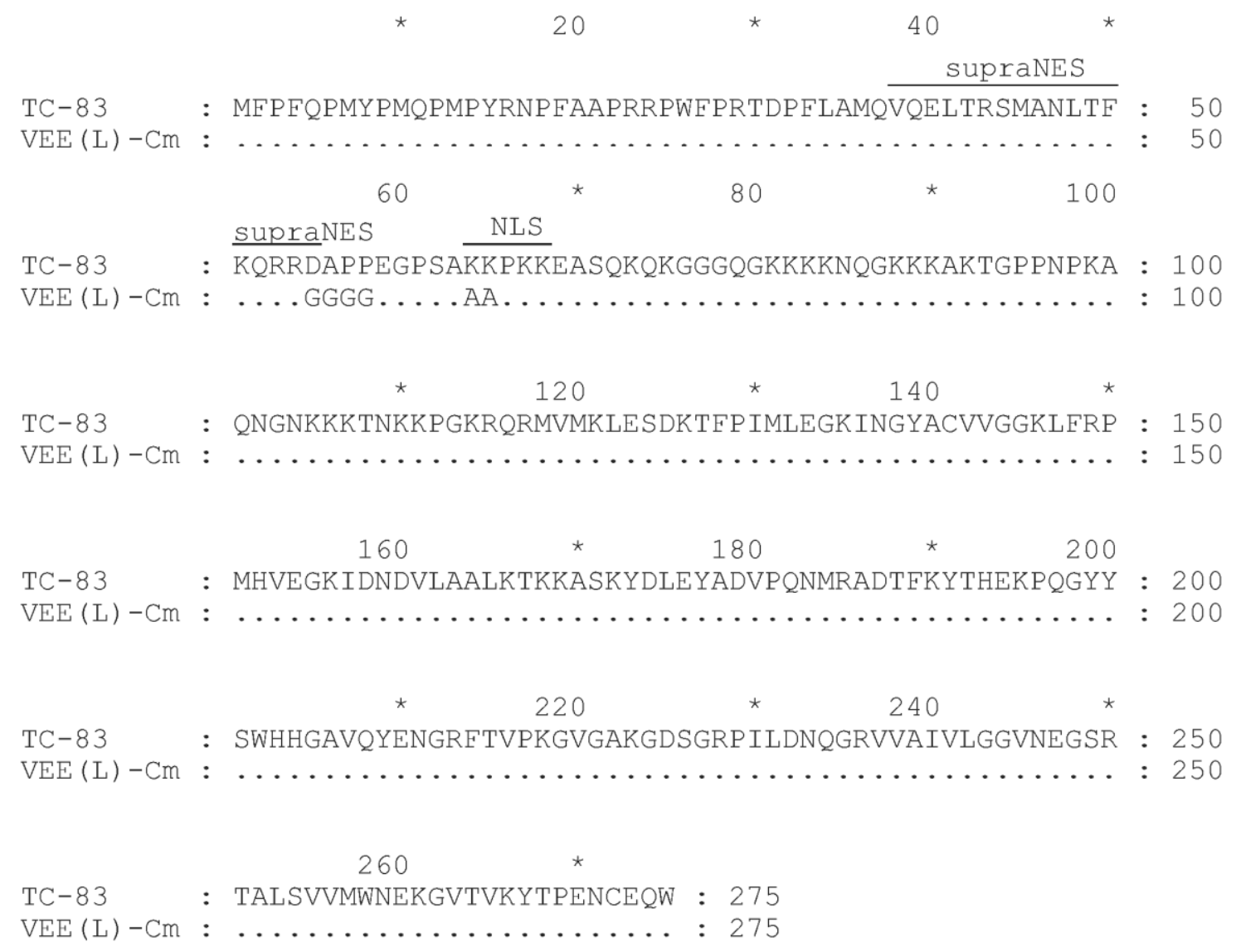

Dots indicate identical amino acid residues. Positions of supraphysiological nuclear export signal (supraNES) and nuclear localization signal (NLS) are outlined.

Fig. 1. Comparison of amino acid sequences of the wild type capsid protein and a mutant capsid protein $(\mathrm{Cm})$ with changes in supraNES and NLS

\section{Gene cassette Pac-2A-GFP}

A gene cassette Pac-2A-GFP contains one long reading frame (ORF) which encodes an enzyme puromycin acetyltransferase (Pac) and a green fluorescent protein (GFP). The amino acid sequences Pac and GFP in a translated polyprotein are separated with a small linker (17 amino acid residues) which is a sequence of autoprotease 2A of a foot-and-mouth disease virus (FMDV). This gene cassette was designed to be cloned into a genome of the non-cytopathic VEE, so that the virus drive intracellular production of the Pac and hence provide cells with a resistance to the antibiotic puromycin, and that the GFP is produced in infected cells which allows visual observance of the virus propagation using a fluorescence microscopy. A nucleotide sequence of the Pac-2A-GFP cassette and the amino acid sequence of the translation product are shown in figure 2. A DNA fragment (1446 bp) comprising the gene cassette was synthesized de novo from oligonucleotides in a manner similar to described in [15]. The fragment was cloned into pGEM-T vector, resulting in the plasmid pPac-2A-GFP. The insert in the pPac-2A-GFP was sequenced and excised for further cloning into the VEE genome. 


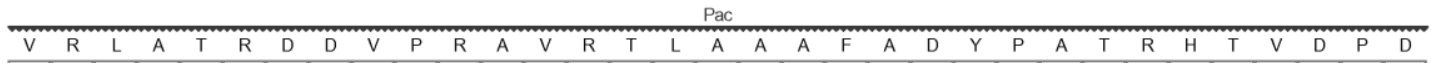

GTGCGCCTCGCCACCCGCGACGACGTCCCCGGGCCGTACGCACCCTCGCCGCCGCGTTCGCCGACTACCCGCCACGCGCCACACCGTCGACCCGGAC

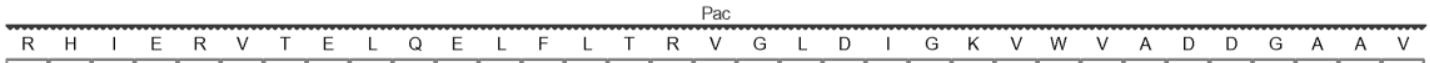

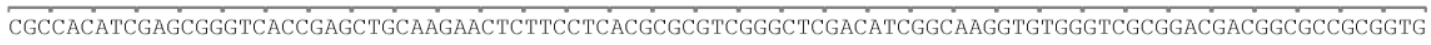

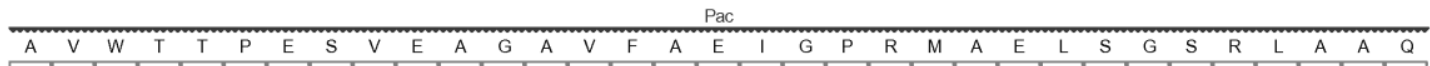
GCGGTCTGGACCACGCCGGAGAGCGTCGAAGCGGGGGCGTGTTCGCCGAGATCGGCCGCGCATGGCCGAGTGAGCGGTCCCGGCTGGCGCGCAG

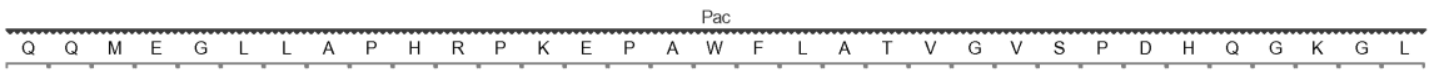
CAACAGATGGAAGGCCTCCTGGCGCCGCACCGGCCAAGGAGCCGCGTGGTCCTGGCCACCGTCGGCGTCTCGCCCGACCACCAGGGCAAGGGTCTG

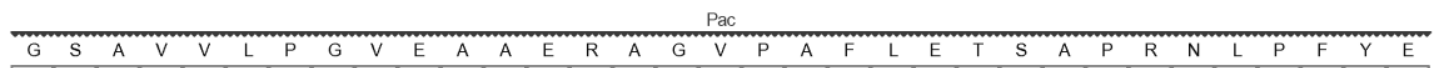
GGCAGCGCCGTCGTGCTCCCCGAGTGGAGGCGGCCGAGCGCGCCGGGGTGCCGCCTTCCTGGAGACCTCCGCGCCCCGCAACCTCCCTTCTACGAG 2A autoprotease (FMDV)

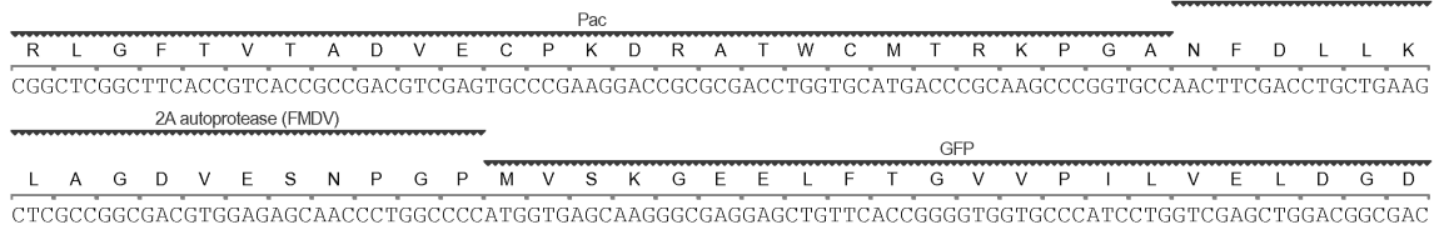

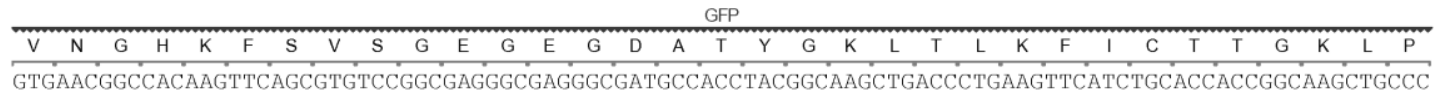

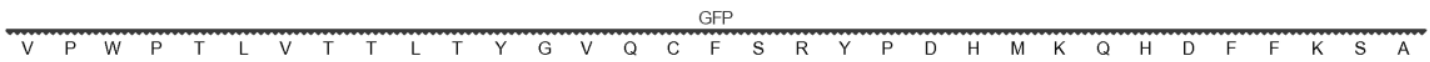
GTGCCCTGGCCCACCCTCGTACCACCCTGACCTACGGCGTGCAGTGCTCAGCCGCTACCCCGACACATGAAGCAGCACGACTTCTTCAAGTCCCC

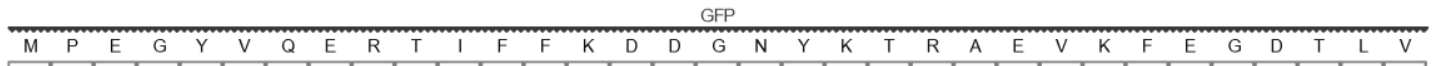
ATGCCCGAAGGCTACGTCCAGGAGCGCACCATCTTCTTCAAGGACGACGGCAACTACAAGACCCGCGCCGAGGTGAAGTTCGAGGGCGACACCCTGGTG

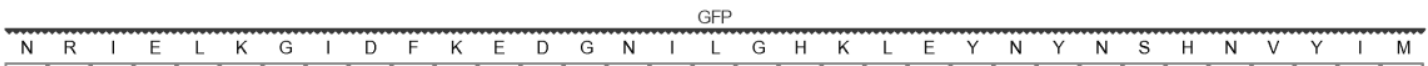

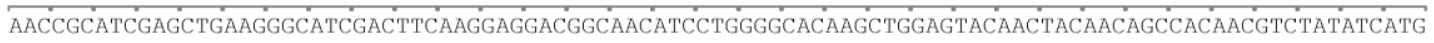

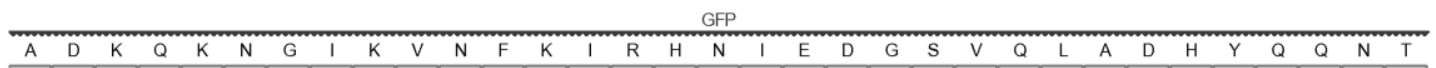
GCCGACAAGCAGAAGAACGGCATCAAGGTGAACTTCAAGATCCGCCACAACATCGAGGACGGCAGCGTGCAGCTCGCCGACCACTACCAGCAGAACACC

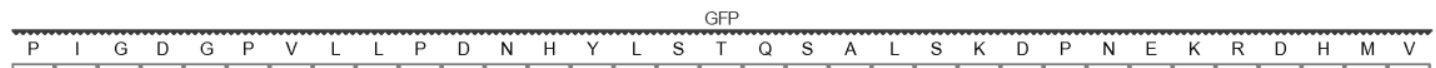

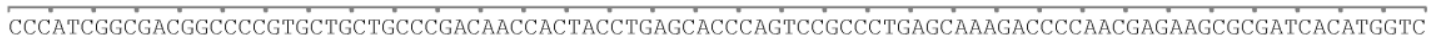
GFP L $\quad$ E $F$ F CTGCTGGAGTCGTGACCGCGCGGATCACTCACGGCATGGACGAGCTGACAATAA

Positions of genes encoding puromycin acetyltransferase (Pac), autoprotease $2 \mathrm{~A}$ of a foot-and-mouth disease virus (FMDV) and GFP, as well as a subgenomic promoter are depicted. Restriction sites used to clone the gene cassette into the VEE genome are shown

Fig. 2. A nucleotide sequence of a gene cassette Pac-2A-GFP

The gene cassette was cloned into the plasmid pVEE(L)-Cm by flanking restriction sites PspOMI and BsrGI. This resulted in a generation of the ultimate construct pVEE(L.Cm).Pac-2A-GFP (Genbank entry: MF136452). The latter plasmid is a molecular clone of a VEE recombinant genome designed to replicate without a CPE and to produce Pac and GFP during an intracellular replication. A genetic map of the VEE(L.Cm).Pac-2A-GFP virus genome is shown in figure 3 . 


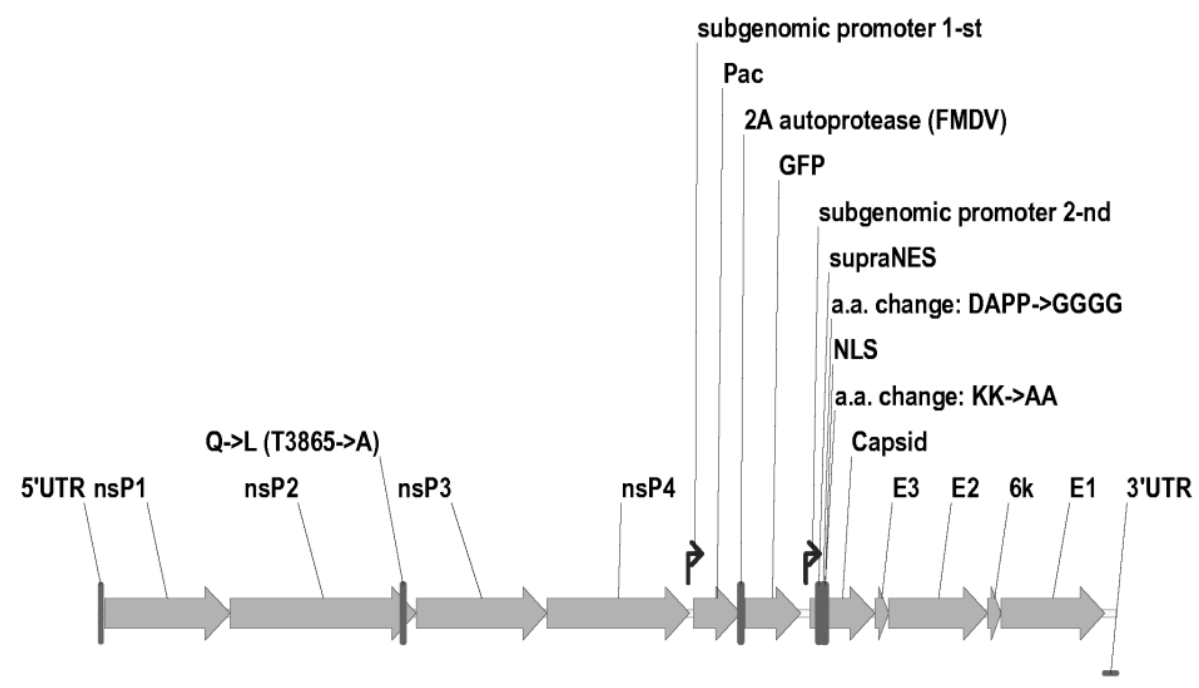

Positions of genes for viral proteins (nsP1-nsP4, capsid, E3-E2-6k-E1), untranslated regions of the genome (UTR), subgenomic promoters (two copies), inserted heterologous genes: Pac (puromycin acetyltransferase), 2A (autoprotease of foot-and-mouth disease virus) and GFP are shown. Positions of changes in nsP2 (Q->L) and capsid protein (DAPP$>$ GGGG, KK->AA) are depicted.

Fig. 3. Map of a genome of a virus VEE(L.Cm).Pac-2A-GFP

\section{Production of virus VEE(L.Cm).Pac-2A-GFP}

Plasmid pVEE(L.Cm).Pac-2A-GFP was linearized by hydrolysis at the unique MluI restriction site which is flanking the 3'end of the viral genome. Viral genomic RNA was synthesized in vitro (results shown in figure 4).

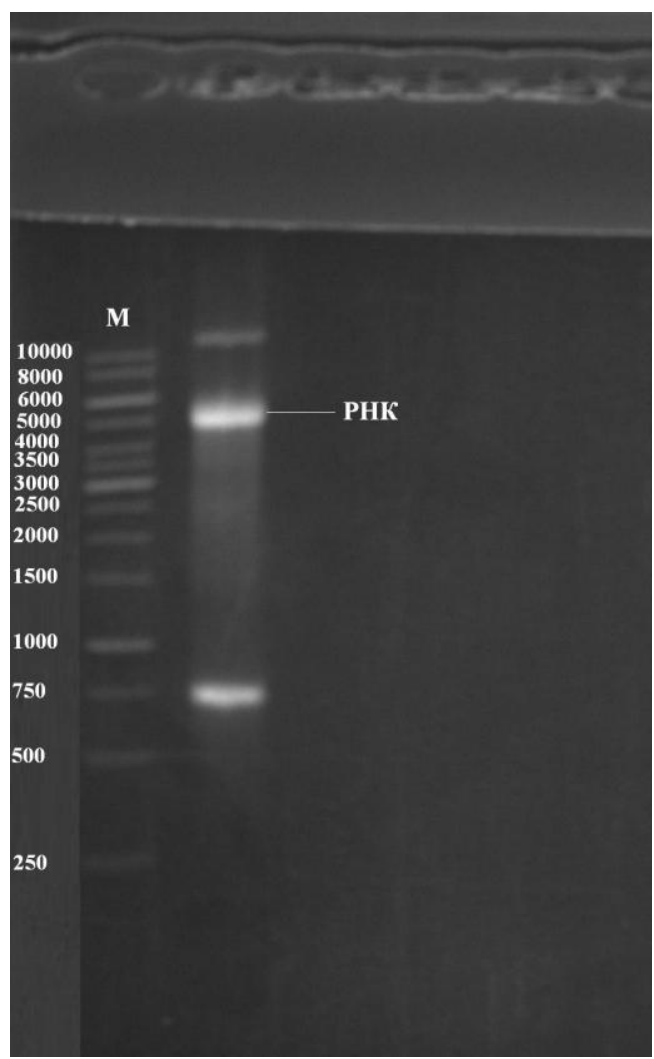

Lane M, marker O'GeneRuler $1 \mathrm{~kb}$ (Fermentas); lane next to the marker, $5 \mu \mathrm{l}$ of the reaction mixture for the in vitro RNA synthesis was applied. The band of the full-length viral genome (12973 nt) is indicated. The RNA band moves in a gel faster than the fragments of the marker with comparable lengths because RNA has a more compact 3D fold compared to DNA. An additional band is visible in the reaction mixture (corresponding to $\sim 750 \mathrm{bp}$ of the marker) which represents a by-product of the in vitro transcription reaction.

Fig. 4. Electrophoresis in $1 \%$ agarose of an in vitro transcription mixture 
Genomic RNAs of viruses with positive-polarity genomes ("positive" polarity means that such RNA is translated on ribosomes) are infectious after entering a cytoplasm. Aliquots of reaction mixtures produced in in vitro transcription reactions $(10 \mu \mathrm{l})$ were transfected into the BHK-21 cells to produce live virus. Twenty-four hours after the transfection almost all cells in the transfected culture demonstrated GFP fluorescence (figure 5). Alphaviruses have rapid replication: the first viral progeny exits from infected or RNA-transfected cells after just 4 hours upon infection or transfection. The transfected culture was monitored for 5 days during which samples of the viruscontaining culture medium were collected. Throughout the observation period the cells in the culture retained a characteristic morphology and continued to divide and ultimately reached overconfluence. With this regard, the virus VEE(L.Cm).Pac-2A-GFP did not show visible signs of a CPE on this (BHK-21) culture. The virus titer in a sample of the medium collected 19 hours after the transfection was $7.2^{*} 10^{\wedge} 7 \mathrm{FFU} / \mathrm{ml}$.

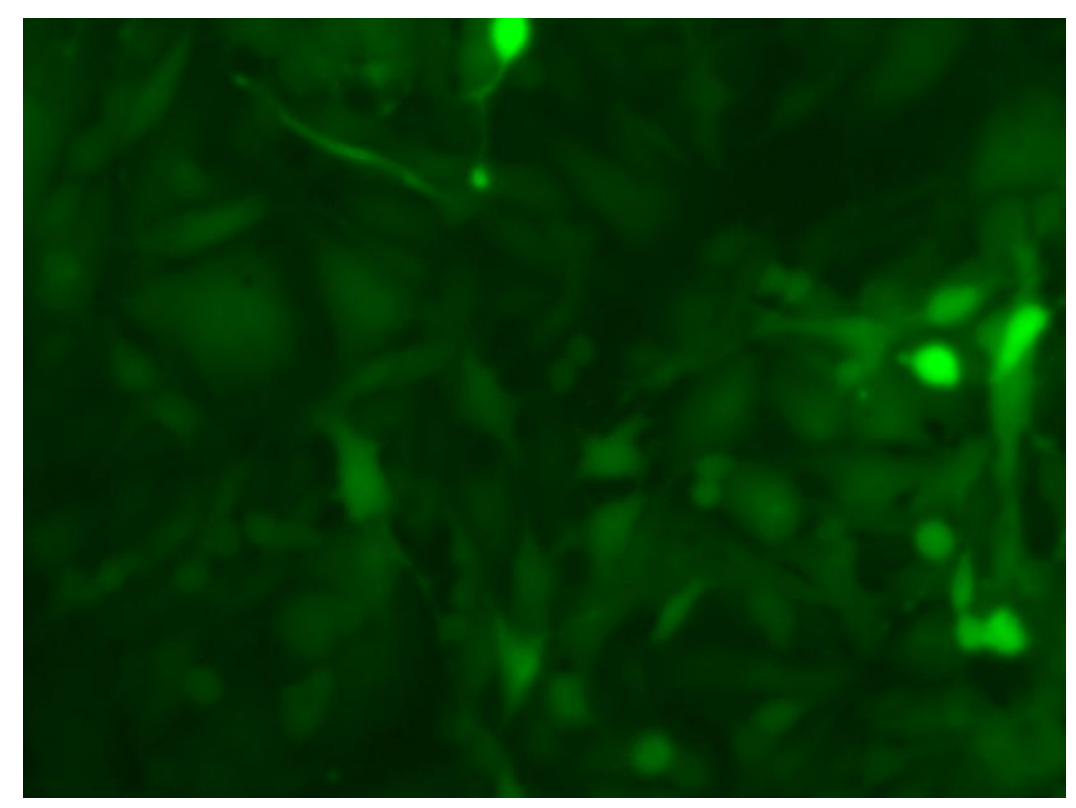

The picture was taken at 24 hours after transfection

Fig. 5. Culture BHK-21 transfected with RNA VEE(L.Cm).Pac-2A-GFP

\section{Growth of VEE (L.Cm).Pac-2A-GFP virus in cell cultures with varying anti-viral resistance}

Growth curve is a graph depicting virus titers in samples taken at various time points after infection. Shapes of the growth curves depend from a multiplicity of infection (MOI), i.e. from a ratio of a number of virus particles (FFU) in an inoculum to counts of cells at the moment of infection. Dynamics of a virus growth differs depending on a species specificity of the cells, and also strongly depends on a differentiation type of the cells (e.g., fibroblasts, epithelial cells, leukocytes, etc.). To characterize the dynamics of growth and to evaluate a CPE of the virus VEE(L.Cm).Pac-2A-GFP, cultures BHK-21, HEK293T and X63-AG8.653 were infected. In this experiment, a single multiplicity of infection was used, MOI=1. One difference in a technique of infecting of the cells with adhesive growth (BHK-21, HEK293T) and plasmacytoma cells X63-AG8.653 (which growth on a substrate and in a suspension) was that a medium was removed from the cultures BHK-21, HEK293T before the infection; upon incubation with the virus inoculum, the inoculum was removed and the complete growing medium (10 ml) was placed into culture vessels. The cells X63-AG8.653 during time from seeding to infection ( $4 \mathrm{~h})$ do not attach to the substrate, so it is impossible to remove the medium from the culture. The culture X63-AG8.653 was infected by an addition of the infectious inoculum directly to the cells in a suspension, using the same MOI=1. In the experiment it was found that on the first day of incubation the infected cells X63-AG8.653 attach to the substrate, and from that time on it is possible to completely replace the medium during a collection of samples. Samples of media were collected from infected cultures every 24 hours, and the media were completely replaced with portions (10 ml) of fresh medium. Propagation of the virus was observed in all cultures. In the culture BHK-21 at 24 hours after the infection, almost all cells were GFP-positive indicating rapid spread of the infection. The cultures differed significantly by an intensity of fluorescence: BHK-21> HEK293T >> X63-AG8.653. The differences in the GFP expression may be attributed to unequal amounts of viral RNA in the cells of various types. Titers of the virus were determined and growth curves were constructed (figure 6). 


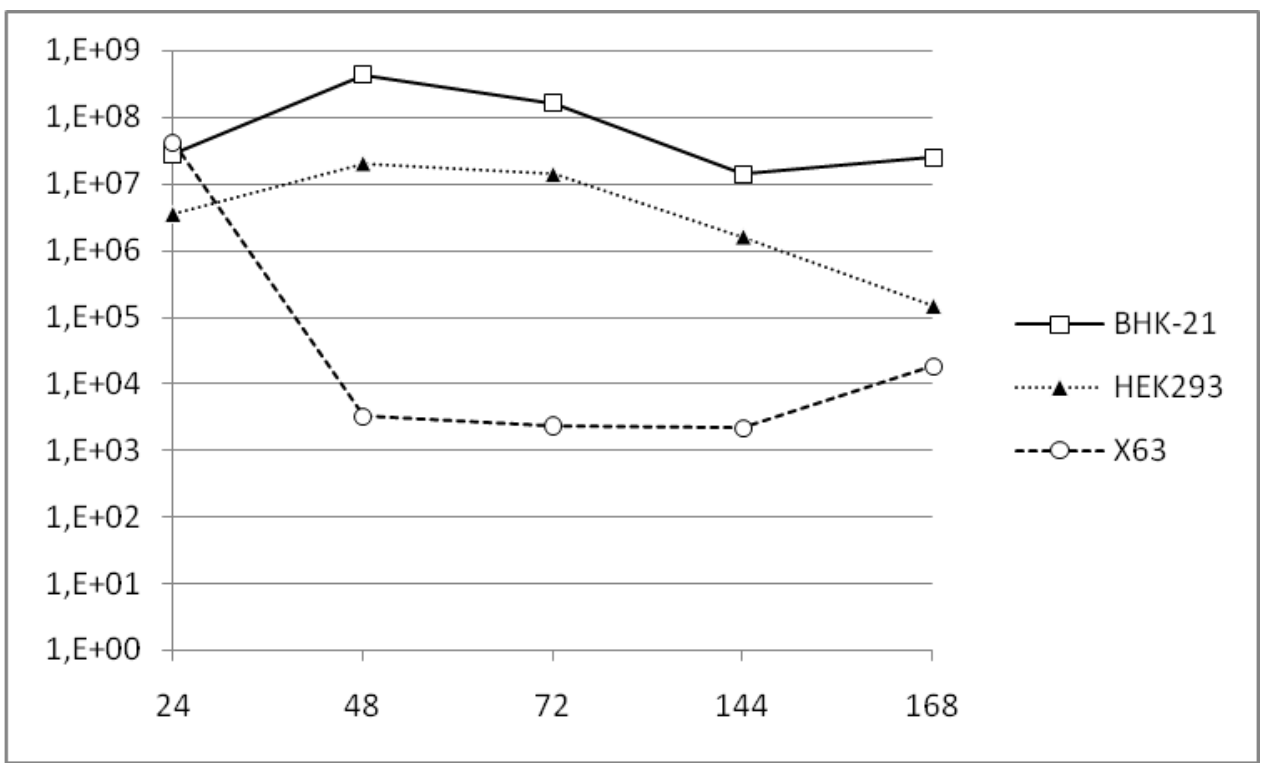

Fig. 6. Growth curves of the virus VEE(L.Cm).Pac-2A-GFP in cultures BHK-21, HEK293T and X63-AG8.653

The BHK-21 cells produce large amounts of the virus: in an acute phase of the infection (48-72 hours after the addition of the virus), the titers exceed $10^{\wedge} 8 \mathrm{FFU} / \mathrm{ml}$. In the culture HEK293T the titers were $\sim 10$ times smaller. The cells X63-AG8.653 showed low production of the virus: the titers during 48-144 hours of observation reached only $\sim 10^{\wedge} 3 \mathrm{FFU} / \mathrm{ml}$. In the X63 culture, a high titer of the virus was detected in the first sample $(24 \mathrm{~h})$, but these are undoubtedly remnants of the virus from the infectious inoculum, since the inoculums could not be removed from this culture until the cells attach ( 24 hours).

Infected cultures continued to grow throughout the experiment, and the cells in the three cultures reached over confluence. In this experiment, the virus VEE(L.Cm).Pac-2A-GFP did not demonstrate CPE in any culture. figure 7 presents photographs of the X63-AG8.653 culture at the end of the acute phase of infection (72 h).

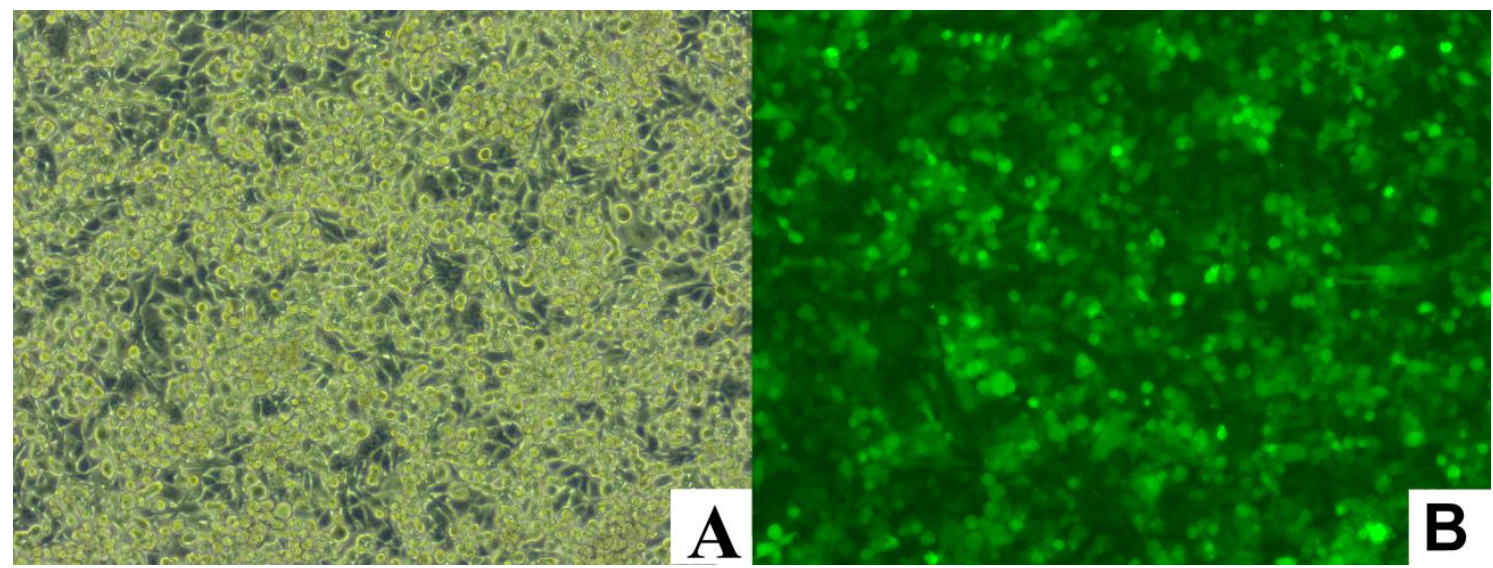

The pictures were taken at 72 hours after infection. Panel A, white light; panel B, in UV light GFP fluorescence is visible

Fig. 7. Culture X63-AG8.653 infected with the virus VEE(L.Cm).Pac-2A-GFP

In this paper, replication of the VEE virus was studied in three cell lines, which are of interest to an industry as producers of recombinant proteins (e.g., biopharmaceuticals). Plasmacytoma cells (including NS0, X63 and others) are routinely used as producers of monoclonal antibodies [21, 22]. Two other cell lines also found application in industrial eukaryotic expression systems: HEK293T (epithelial kidney cells of the human embryo) [23] and BHK-21 (golden hamster kidney fibroblasts) [24].

The cell cultures used in this study show different abilities to withstand viral infection. One of the most studied mechanisms of intracellular innate immunity is an establishment of an antiviral state under an influence of interferons (IFNs). Efficacious suppression of replication of RNA viruses in cells with undisturbed natural mechanisms of antiviral response is a problem for utilization of alphavirus vectors with a number of cell lines known for a high production, for example, cells from myeloid or lymphoid origin. IFNs are a heterogeneous family 
of secreted glycoproteins which include several types. The IFN type I includes IFN-alpha, -beta, -delta and -cappa $[25,26]$, type II is the IFN-gamma, and type III is the IFN-lambda. The IFNs are signaling molecules, they are secreted from the infected cells and provide autocrine regulation (i.e. on the cell which itself produces the IFN) and paracrine regulation (on neighboring uninfected cells). On plasma membranes, the IFNs interact with specific receptors; each type of the IFN has a type-specific receptor (IFNAR for the type I IFNs, IFNGR for type II, IL-28Ralpha/IL-10R-beta for type III). Binding of an IFN to its receptor signals via the JAK-STAT pathway and activates a large number of genes in nuclear DNA (so-called interferon-stimulated genes, ISG). Various intracellular factors are synthesized from the activated ISGs, including protein kinase PKR, 2',5'-oligoadenylate synthetase, RNase L and GTPases of Mx proteins. The ultimate result of the IFN action is a degradation of intracellular double-stranded RNAs (replicative intermediates of RNA viruses), an inhibition of translation and an establishment of a so-called "antiviral state". The antiviral state presents itself as an insensitivity of naïve (uninfected) cells to the infection. The induction of the antiviral state is considered to be the main (although not the only) way by which the cells of vertebrates limit the replication of viruses. Alphaviruses are considered to be very sensitive to the antiviral effects of the type I IFNs [27, 28, 29, 30].

BHK-21 cells are defective in production of the type I IFNs. BHK-21 do not produce IFN-alpha and IFN-beta and thus are unable to establish the antiviral state because of an endogenous interferon [31]. This may be an explanation of the highest titers of the virus in the BHK-21 culture $\left(\sim 10^{\wedge} 8 \mathrm{FFU} / \mathrm{ml}\right)$. Other explanations are possible and include, for example, higher infectivity of the VEE virions to BHK-21 cells due to a greater density or higher affinity of cellular receptors on these cells. The culture HEK293T responds to a viral infection by an induction of the endogenous IFN and activation of ISGs [32].

Cells of a myeloid or lymphoid origin (such as the plasmacytoma cells X63-AG8.653) have a strong ability to recognize infection by viruses. The recognition of infection occurs with a participation of pattern recognition receptors (PRR) located on a plasma membrane or on membranes of the endosomes. This is a fundamental feature of the myeloid and lymphoid differentiation types, which include regulatory cells of the immune system (derivatives from a myeloid precursor are macrophages and dendritic cells; products of lymphoid pathway are B- and Tlymphocytes) [33]. The infected culture X63-AG8.653 produces the VEE virus to titers 100,000 times lower than the BHK-21 culture. This observation demonstrates a work of the mechanism which effectively limits the replication of RNA viruses. For possible practical utilization of the VEE vector it is important that the VEE is still able to induce a persistent infection in the X63-AG8.653 cells. This ability to infect cells of the myeloid origin is not typical for representatives of the genus Alphavirus. Majority of species in this genus, including a closely related Eastern equine encephalitis virus (EEE) are unable to infect the myeloid cells. It is believed that the avoidance of effector cells of the immune system allows the virus to reduce an activation of the immune system, and diminish a production of the IFNs in tissues, thus lowering an intensity of the organism's response to the infection [34,35]. The plasmacytoma cells (X63, NS0, Sp2/0 and others) are producers of secreted proteins with the highest described levels of production among all cell types. The ability of the VEE to infect the plasmacytoma cell lines paves the way to create new eukaryotic expression systems with an appealing expected productivity.

\section{CONCLUSION}

Further studies with the described experimental system will allow deepening knowledge about the mechanisms of viral resistance to interferons. The virus described herein can be used as an effective vector for the expression of recombinant proteins in mammalian, bird and insect cells. It is possible to use this viral vector in a biotechnology industry. The ability to accumulate to high titers and non-cytopathic replication are remarkable properties which distinguish the described virus from natural strains of alphaviruses. Genetic engineering of RNA viruses, in particular alphaviruses, expands the toolbox for molecular biology and biotechnology.

\section{Funding}

This work was supported by a funding from Ministry of Education and Science of the Republic of Kazakhstan by grant No. 0214RK02428.

\section{REFERENCES}

1. De Jesus M., Wurm F.M. Manufacturing recombinant proteins in kg-ton quantities using animal cells in bioreactors. Eur. J. Pharm. Biopharm, 2011, no. 78, pp. 184-188.

2. Baldi L., Hacker D.L., Adam M., Wurm F.M. Recombinant protein production by large-scale transient gene expression in mammalian cells: state of the art and future perspectives. Biotechnol. Lett., 2007, no. 29, pp. 677-684.

3. Luo J., Deng Z.L., Luo X., Tang N., et al. A protocol for rapid generation of recombinant adenoviruses using the AdEasy system. Nat. Protoc., 2007, no. 2, pp. 1236-1247. 
4. Schott J.W., Hoffmann D., Schambach A. Retrovirus-based vectors for transient and permanent cell modification. Curr. Opin. Pharmacol, 2015, no. 24, pp. 135-146.

5. Farazmandfar T., Khanahmad Shahreza H., Haghshenas M.R., Janbabai G., Azadeh H., Mansour Samaei N. Use of integrase-minus lentiviral vector for transient expression. Cell J., 2012, no. 14, pp. 76-81.

6. Kremer M., Volz A., Kreijtz J.H., Fux R., Lehmann M.H., Sutter G. MVA Methods. Methods of working with recombinant vaccinia virus. Biol., 2012, no. 890, pp. 59-92.

7. Lackner A., Kreidl E., Peter-Vorosmarty B., Spiegl-Kreinecker S., Berger W., Grusch M. Stable protein expression in mammalian cells using baculoviruses. Methods Mol. Biol., 2012, no. 801, pp. 75-92.

8. Aranda A., Ruiz-Guillen M., Quetglas J.I., Bezunartea J., Casales E., Smerdou C. Recent patents on alphavirus protein expression and vector production. Recent Pat. Biotechnol., 2011, no. 5, pp. 212-226.

9. Casales E., Aranda A., Quetglas J.I., et al. A novel system for the production of high levels of the functional proteins in a stable cell with a Semliki Forest virus noncytopathic vector. N. Biotechnol., 2010, no. 27, pp. $138-148$.

10. Lundstrom K. Expression of the mammalian membrane in mammalian cells using Semliki Forest virus vectors. Methods Mol. Biol., 2010, no. 601, pp. 149-163.

11. Nivitchanyong T., Tsai Y.C., Betenbaugh M.J., Oyler G.A. An improved in vitro and in vivo Sindbis virus expression system through host and virus engineering. Virus Res., 2009, no. 141, pp. 1-12.

12. Gehrke R., Heinz F.X., Davis N.L., Mandl C.W. Heterologous gene expression by infectious and replicon derived from tick-borne encephalitis virus and direct comparison of this flavivirus system with an alphavirus replicon. J. Gen. Virol., 2005, no. 86, pp. 1045-1053.

13. Yoshioka N., Gros E., Li H,R., et al. Efficient generation of human iPSCs by a synthetic self-replicative RNA. Cell Stem Cell., 2013, no. 13, pp. 246-254.

14. Lundstrom K. Semliki Forest virus vectors for rapid and high-level expression of integral membrane proteins. Biochim. Biophys. Acta, 2003, no. 1610, pp. 90-96.

15. Kim Y.G., Baltabekova A.Z., Zhiyenbay E.E., et al. Recombinant Vaccinia virus-coded interferon inhibitor B18R: Expression, refolding and a use in a mammalian expression system with a RNA-vector. PLoS One, 2017 , no. 12.

16. Ramakrishnan M.A. Determination of 50\% endpoint titer using a simple formula. World J. Virol., 2016 , no. 5, pp. 85-86.

17. Petrakova O., Volkova E., Gorchakov R., Paessler S., Kinney R.M., Frolov I. Noncytopathic replication of Venezuelan equine encephalitis virus and eastern equine encephalitis virus replicons in Mammalian cells. J. Virol., 2005, no. 79, pp. 7697-7608.

18. Atasheva S., Kim D.Y., Frolova E.I., Frolov I. Venezuelan equine encephalitis, virus variants, lacking transcription inhibitory functions, highly attenuated phenotype. J Virol., 2015, no. 89, pp. 71-82.

19. Garmashova N., Atasheva S., Kang W., Weaver S.C., Frolova E., Frolov I. Analysis of Venezuelan equine encephalitis. J Virol., 2007, no. 81, pp. 13552-13565.

20. Atasheva S., Fish A., Fornerod M., Frolova E.I. Venezuelan equine Encephalitis virus capsid protein forms a tetrameric complex with CRM1 and importin alpha / beta that obstructs the nuclear pore complex function. $J$. Virol., 2010, no. 84, pp. 4158-4171.

21. Barnes L.M., Bentley C.M., Dickson A.J. Characterization of the stability of recombinant protein production in the GS-NS0 expression system. Biotechnol. Bioeng., 2001, no. 73, pp. 261-270.

22. Bebbington C.R., Renner G., Thomson S., King D., Abrams D., Yarranton G.T. High-level expression of a recombinant antibody from myeloma cells using a glutamine synthetase gene as an amplifiable selectable marker. Biotechnology (NY), 1992, no. 10, pp. 169-175.

23. Baldi L., Muller N., Picasso S., et al. Transient gene expression in suspension HEK-293 cells: application to large-scale protein production. Biotechnol Prog., 2005, no. 21, pp. 148-153.

24. Wurm F.M. Production of recombinant protein therapeutics in cultivated mammalian cells. Nat. Biotechnol., 2004, no. 22, pp. 1393-1398.

25. Kawaguchi S., Ishiguro Y., Imaizumi T., et al. Retinoic acid-inducible gene-I is constitutively expressed and involved in IFN-gamma-stimulated CXCL9-11 production in the intestinal epithelial cells. Immunol. Lett., 2009, no. 123, pp. 9-13.

26. Randall R.E., Goodbourn S. Interferons and viruses: an interplay between induction, signalling, antiviral responses and virus countermeasures. J. Gen. Virol., 2008, no. 89, pp. 1-47.

27. Zhang Y., Burke C.W., Ryman K.D., Klimstra W.B. Identification and characterization of interferoninduced proteins that inhibit alphavirus replication. J. Virol., 2007, no. 81, pp. 11246-11255.

28. Karki S., Li M.M., Schoggins J.W., Tian S., Rice C.M., MacDonald M.R. Multiple interferon stimulated genes synergize with the zinc finger antiviral protein to mediate anti-alphavirus activity. PLoS One, 2012, no. 7.

29. Hyde J.L., Gardner C.L., Kimura T., et al. A viral RNA structural element alters host recognition of nonself RNA. Science, 2014, no. 343, pp. 783-787.

30. Poddar S., Hyde J.L., Gorman M.J., Farzan M., Diamond M.S. The Interferon-Stimulated Gene IFITM3 Restricts Infection and Pathogenesis of Arthritogenic and Encephalitic Alphaviruses. J. Virol., 2016, no. 90, pp. 8780-8794. 
31. Spotts D.R., Reich R.M., Kalkhan M.A., Kinney R.M., Roehrig J.T. Resistance to alpha / beta interferons correlates with the epizootic and virulence potential of Venezuelan equine encephalitis viruses and is determined by the 5 'noncoding region and glycoproteins. J. Virol., 1998, no. 72, pp. 10286-10291.

32. Reynolds A., Anderson E.M., Vermeulen A., et al. Induction of the interferon response by siRNA is cell type- and duplex length-dependent. RNA, 2006, no. 12, pp. 988-993.

33. McNab F., Mayer-Barber K., Sher A., Wack A., O'Garra A. Type I interferons in infectious disease. Nat. Rev. Immunol., 2015, no. 15, pp. 87-103.

34. Ryman K.D., Klimstra W.B. Host responses to alphavirus infection. Immunol. Rev., 2008, no. 225, pp. $27-$ 45 .

35. Alsharifi M., Lobigs M., Regner M., Lee E., Koskinen A., Mullbacher A. Type I interferons trigger systemic, partial lymphocyte activation in response to viral infection. J. Immunol., 2005, no. 175, pp. 4635-4640. 